\section{Die Ferratgleichgewichte in Alkalihydroxydschmelzen}

Von Hermann Lux und Titus Niedermaier

Anorganisch-chemisches Laboratorium der TH München und Institut für anorganische Chemie der Universität München

(Z. Naturforschg. 11 a, 762 [1956] ; eingegangen am 24. Juli 1956)

Nach einem Verfahren, das kürzlich von uns beschrieben worden ist ${ }^{1}$, lassen sich Extinktionsmessungen an aggressiven Schmelzen oder anderen Flüssigkeiten auch dann durchführen, wenn kein durchsichtiges Küvettenmaterial zur Verfügung steht. Durch Aufnahme der Extinktionskurven verdünnter Ferratlösungen in Alkalihydroxydschmelzen bei $300-500^{\circ} \mathrm{C}$ unter definierten Sauerstoff- und Wasserdampfpartialdrucken hat sich ergeben, daß das rotviolette Fer$\operatorname{rat}(\mathrm{VI})$ in $\mathrm{KOH}$-Schmelzen bei $310^{\circ} \mathrm{C}$ in Sauerstoff völlig stabil ist. Sein Anteil im Gleichgewicht erreicht bei kleinen Wasserdampfpartialdrucken fast $100 \mathrm{Mol}$ -

1 H. Lux u. T. Niedermaier, Z. anorg. allg. Chem. 285, 246 [1956].

\section{Messung der Oszillatorenstärke der Eisen- resonanzlinie $3720 \AA ̊$ aus der Abklingdauer}

\author{
Von O. Osberghaus und K. Ziock
}

Physikalisches Institut der Universität Bonn

(Z. Naturforschg. 11 a, 762-763 [1956]; eingegangen am 2. August 1956)

Die Oszillatorenstärke der Eisenresonanzlinie $3720 \AA$ ist bisher von zwei Autoren ${ }^{1,2}$ nach zwei verschiedenen Absorptionsverfahren gemessen worden. KING fand für sie den Wert $f=0,013$, entsprechend einer Abklingkonstanten von $5 \cdot 10^{6} \mathrm{sec}^{-1}$, während Kopfermann und WESSEL $f=0,043$, entsprechend einer Abklingkonstanten von $1,7 \cdot 10^{7}$, gemessen haben. Diese Diskrepanz gab Veranlassung zu einer Neubestimmung der Oszillatorenstärke nach einem völlig anderen Verfahren.

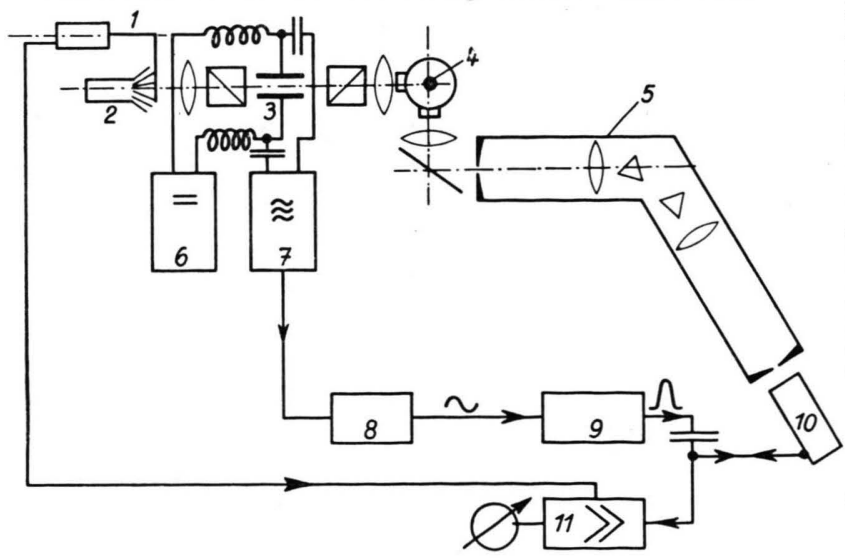

Abb. 1. Meßanordnung. prozent. Bei geringerem Sauerstoffdruck oder höherem Wasserdampfdruck geht der Anteil von Ferrat(VI) zurück; dafür beteiligt sich ein gelblich-braunes Ferrat(III) und ein farbloses Ferrat(III) an dem stark von der Temperatur abhängigen Gleichgewicht.

Wie bereits bei Untersuchungen über die Manganatgleichgewichte und über die Peroxydgleichgewichte von uns festgestellt werden konnte, verhalten sich $\mathrm{NaOH}$ Schmelzen völlig anders als KOH-Schmelzen. Dasselbe zeigt sich bei den Ferratgleichgewichten. Ferrat(VI) tritt in $\mathrm{NaOH}-\mathrm{Schmelzen}$ nicht auf. Die quantitative Lösungen ein Ferrat vorliegt, dessen Menge vom Untersuchung hat aber gezeigt, daß auch in diesen Sauerstoffpartialdruck abhängt und in reinem Sauerstoff bei $410^{\circ} \mathrm{C}$ sogar stark überwiegt, während in reinem Stickstoff nur ganz wenig $\mathrm{Fe}_{2} \mathrm{O}_{3}$ in Lösung geht. Das Ferrat ist braun, ähnlich wie Ferrat(III) ; seine Extinktionskurve verläuft jedoch merklich steiler. Die tiefbraunen Lösungen von $\mathrm{Fe}_{2} \mathrm{O}_{3}$ in $\mathrm{NaOH}$-Schmelzen enthalten somit nicht, wie man bisher annahm, das Eisen in dreiwertiger Form, sondern in einer höheren Oxydationsstufe. $\mathrm{Ob}$ es sich dabei um Ferrat(IV) oder Ferrat(V) handelt, konnte noch nicht entschieden werden.

Strahlt man Licht einer Resonanzlinie des Eisens, dessen Intensität moduliert ist, in Eisendampf ein, so ist das Streulicht ebenfalls moduliert. Bei vorgegebener Abklingkonstante des untersuchten Übergangs ist die Modulation des Streulichts in berechenbarer Weise gegenüber der des eingestrahlten Lichtes verzögert und in ihrer Form verändert. Die Messung der Form und der zeitlichen Verzögerung der Streulichtimpulse gibt also Aufschluß über die gesuchte Abklingkonstante.

Die Meßanordnung zeigt Abb. 1. Als Lichtquelle dient eine Glimmentladung in der Eisenhohlkathode (2). Zwischen Lichtquelle und Eisendampfstrahl (4) wird das Licht mit Hilfe der zwischen zwei Nicolschen Prismen angeordneten Kerr-Zelle (3) moduliert. Die Modulationsfrequenz ist $1 \mathrm{MHz}$. Mit dem auch im UV durchsichtigen $o$-Dichlorbenzol als KERR-Flüssigkeit läßt sich bei $8 \mathrm{~mm}$ Plattenabstand und $50 \mathrm{~mm}$ Plattenlänge der Kerr-Zelle 100-proz. Modulation erreichen, wenn an die Zelle eine Wechselspannung von $15 \mathrm{kV}$ und zusätzsich eine Gleichspannung von dem gleichen Betrag angelegt wird. Aus dem vom Eisendampfstrahl gestreuten Licht trennt ein Quarzspektrograph (5) die zu untersuchende Linie $3720 \AA ̊$ heraus. Zum Nachweis des Resonanzlichtes dient ein Photomultiplier EMI 5311 (10). Die Anode dieses Photomultipliers wird über einen Impulsgenerator (9) mit der Frequenz des die KerRZelle erregenden Senders (7) getastet, so daß der Photomultiplier immer nur während dieser Hochfrequenzimpulse für das ankommende Licht empfindlich ist. Der Impulsgenerator wird vom Sender über eine veränderliche Laufzeitstrecke (8) gesteuert, die es ge stattet, die abgegebenen Impulse in 32 Stufen um ins-

1 R. B. KING, Astrophys. J. 95, 78 [1941].

2 H. Kopfermann u. G. Wessel, Z. Phys. 130, 100 [1951]. 